\title{
Sonication-assisted dispersion of carbon nanotubes in aqueous solutions of the anionic surfactant SDBS: The role of sonication energy
}

\author{
YANG Kun ${ }^{1,2^{*}}$, YI ZiLi ${ }^{1,2}$, JING QingFeng $^{1,2}$, YUE RenLiang $^{3}$, JIANG Wei $^{4} \&$ LIN DaoHui $^{1,2}$ \\ ${ }^{1}$ Department of Environmental Science, Zhejiang University, Hangzhou 310058, China; \\ ${ }^{2}$ Zhejiang Provincial Key Laboratory of Organic Pollution Process and Control, Hangzhou 310058, China; \\ ${ }^{3}$ Institute of Process Engineering, Chinese Academy of Sciences, Beijing 100190, China; \\ ${ }^{4}$ Environment Research Institute, Shandong University, Jinan 250100, China
}

Received August 27, 2012; accepted December 25, 2012; published online February 25, 2013

\begin{abstract}
Sonication is a powerful technique to promote the dispersion of carbon nanotubes (CNTs) and enhance their solubility; this is necessary for CNT applications, especially in the biochemical and biomedical fields. In this study, batch experiments were conducted to evaluate the role of sonication energy on the dispersion of CNTs in the presence of a widely used anionic surfactant, sodium dodecylbenzene sulfonate (SDBS). It was observed that the concentration of dispersed CNTs in the SDBS solution depended on the sonication energy, but not the sonication time or output power of the sonicator alone. The amount of dispersed CNTs was positively correlated with the concentrations of SDBS and CNTs, and the length of the CNTs. The promotion of oxygen-containing functional groups on the dispersed CNTs was observed at relatively low sonication energies. The optimal energy, i.e. the minimum energy supplied by sonication to achieve a saturated suspension of dispersed CNTs in the SDBS solution, was CNT diameter-dependent, because of the larger vdW forces between tubes of smaller diameter. An exponential decay curve was constructed for the optimal energy values as a function of the outer CNT diameter, to assist in determining the energy needed to disperse CNTs.
\end{abstract}

carbon nanotubes, sonication energy, dispersion, surfactant

Citation: Yang K, Yi Z L, Jing Q F, et al. Sonication-assisted dispersion of carbon nanotubes in aqueous solutions of the anionic surfactant SDBS: The role of sonication energy. Chin Sci Bull, 2013, 58: 2082-2090, doi: 10.1007/s11434-013-5697-2

Carbon nanotubes (CNTs), i.e. single-walled carbon nanotubes (SWCNTs) and multi-walled carbon nanotubes (MWCNTs), have attracted special attention for their potential applications in catalyst supports, optical devices, quantum computers, and the biomedical field, because of their outstanding physical and chemical properties [1,2]. However, the poor solubility of CNTs in water-aggregates form because of the highly attractive van der Waals (vdW) forces (e.g. $500 \mathrm{eV} / \mu \mathrm{m}$ of tube length for SWCNTs) between tubes-significantly limits their applications [3,4].

In the past decade, extensive efforts have been made to develop techniques to disperse CNTs in water. These tech-

*Corresponding author (email: kyang@zju.edu.cn) niques can be generally categorized into covalent functionalization and noncovalent functionalization [5]. Covalent functionalization involves the oxidative formation of carboxyl functional groups on the CNT surface, followed by the grafting of organic moieties on the surface [5]. However, the application of covalent functionalization techniques is limited by the fact that they alter the inherent electrical, mechanical, and optical properties of CNTs [6]. Noncovalent functionalization uses dispersants such as surfactants and organic solvents to promote CNT dispersion, leaving the electronic structures and properties of the CNTs unaltered. However, evidence has shown that CNTs dispersed using organic solvents are generally incompatible with living organisms [7]. CNTs dispersed using surfactants, on the 
other hand, are more compatible with living organisms, and have therefore been applied in the fields of biochemistry and biomedical engineering [5]. The surfactants used include cetyltrimethylammonium bromide [8], Triton X-100 [9], and sodium dodecylbenzene sulfonate (SDBS) $[8,10]$. Among these surfactants, SDBS, an anionic surfactant, is outstanding, because of its negative charge and benzene ring [10]. However, surfactant-assisted CNT dispersion cannot occur spontaneously. Sonication is a mechanical method that is frequently employed in combination with surfactants to break, disperse, or de-bundle CNT aggregates or bundles in the dispersing process [11].

Studies $[7,11]$ have shown that, within limits, the longer the sonication time, the better the CNT suspension and the smaller the suspended CNT particle size; both of these properties reach an equilibrium value with further sonication. The sonication time was therefore suggested as a parameter to identify the degree of CNT dispersion $[7,11]$. However, other researchers [11-14] observed that the dispersion degree of a given CNT suspension depended on the sonication energy, rather than the sonication time. The sonication energy $(E, \mathrm{~J} / \mathrm{mL})$, which is defined using the following equation, was therefore used to identify the degree of CNT dispersion [13]:

$$
E=P \times t / V,
$$

where $P$ is output power of the sonicator, $\mathrm{W} ; t$ is the sonication time, in $\mathrm{s}$; and $V$ is the total volume of liquid sonicated by the sonicator, in $\mathrm{mL}$. These results indicated that an insufficient supply of sonication energy could lead to the insufficient dispersion of CNTs, and dispersed CNT particles that are not small enough $[12,13]$, while excessive sonication energy would not be economical. In previous studies, the sonication energies $(E)$ applied to disperse CNTs in surfactant solutions ranged from 320 to $50400 \mathrm{~J} / \mathrm{mL}$, with the sonication time $(t)$ ranging from 5 to $120 \mathrm{~min}$ (Table 1) $[11,12,15-20]$. It is therefore necessary to examine the role of the sonication energy in surfactant-assisted CNT dispersion, to facilitate the effective use of energy in the industrial applications of CNTs. In particular, the influence of surfac- tant concentration, as well as the concentration, diameter, length, and surface functional groups of CNTs should be examined, because these factors can significantly affect the CNT dispersion [12,21-23]. The optimal energy is defined here as the minimum sonication energy needed to obtain a saturated suspension of CNTs in the surfactant solution. Prior to the application of the sonication energy as a parameter to identify the degree of CNT suspension, it should also be determined whether the same quantity of energy supplied by different processes (e.g. high output power with short time, or low output power with long time) would result in the same degree of CNT dispersion.

In this study, batch experiments were conducted to elucidate the role of sonication energy, using the widely used anionic surfactant SDBS in CNT dispersions. The influence of the sonication time, the output power of the sonicator, the SDBS concentration, the CNT concentration, the CNT diameter/length, and the surface functional groups of the CNTs on the degree of dispersion and the optimal energy were examined. The results of this study will be useful in facilitating the effective use of energy in industrial CNT applications. The results will also be essential for environmental risk assessments for CNTs and organic contaminants, because (1) dispersed CNTs can easily move long distances in the environment [25-28], and (2) surfactants, which are widely used in cleaning, industrial, agricultural, and personal care products, and are distributed in the natural environment $[29,30]$, have the ability not only to disperse CNTs, but also to alter the sorption, transport, and fate of organic contaminants in the environment $[27,31]$.

\section{Materials and methods}

\subsection{Materials}

SDBS was purchased from Tokyo Chemical Industry Co., Ltd., and was used as a dispersant without further treatment. Four SWCNT samples-purified SWCNTs (P-SWCNTs), shortened SWCNTs (S-SWCNTs), hydroxylated SWCNTs (H-SWCNTs), and carboxylated SWCNTs (C-SWCNTs)-

Table 1 Experimental data on dispersing CNTs in aqueous phase with the aid of surfactants ${ }^{\text {a) }}$

\begin{tabular}{|c|c|c|c|c|c|c|c|c|}
\hline Materials & Length $(\mu \mathrm{m})$ & $O D(\mathrm{~nm})$ & Surfactant & $C(\mathrm{mg} / \mathrm{L})$ & $T(\min )$ & Power $(\mathrm{W})$ & Energy $(\mathrm{J} / \mathrm{mL})$ & Reference \\
\hline MWCNTs & $1-2$ & 28 & SDBS, CTAB, TX-100 & 40 & 90 & 100 & 540 & {$[15]$} \\
\hline MWCNTs & - & $10-20$ & SDS & $100-4000$ & 50 & 20 & 3000 & [11] \\
\hline MWCNTs & $0.1-10$ & 10 & SDBS; SDS & 1000 & 120 & 40 & 8228 & [16] \\
\hline MWCNTs & $0.5-40$ & $10-20$ & GA & 2500 & 40 & 130 & 624 & [12] \\
\hline SWCNTs & - & $0.7-1.1$ & SDS & 10000 & 10 & 540 & 1620 & [20] \\
\hline SWCNTs & - & $<1$ & SDBS; CTAB; TX-100 & 1000 & 120 & 165 & 50400 & [24] \\
\hline SWCNTs & - & 0.9 & SDBS; SDS; NaC & 10000 & 30 & 10 & 3000 & [18] \\
\hline SWCNTs & - & $0.8-1.3$ & SDBS; DOC & 10000 & 8 & $20-25$ & $320-400$ & [23] \\
\hline SWCNTs & 1 & $0.7-1.4$ & SDS & $200-10000$ & $6-7$ & 20 & $720-840$ & [19] \\
\hline
\end{tabular}

a) $C$, added dose of surfactants; $O D$, outer diameter of CNTs; $T$, sonication time; -, data not available. 
and three MWCNT samples with various outer diameters (ODs)-MWCNT30 with OD of 20-30 nm, MWCNT50 with OD of $30-50 \mathrm{~nm}$, and MWCNT100 with OD of 60-100 nm-were purchased from Chengdu Organic Chemistry Ltd. SEM images of the P-SWCNTs and three MWCNTs are shown in Figure S1. S-SWCNTs were produced via mechanical cutting of the P-SWCNTs. HSWCNTs and C-SWCNTs were produced by oxidizing the P-SWCNTs with $\mathrm{KMnO}_{4}$ in an $\mathrm{H}_{2} \mathrm{SO}_{4}$ solution. Selected properties of these CNTs are listed in Table 2.

\subsection{Characterization of CNTs}

The specific surface area and pore volume distribution of the CNTs were calculated from $\mathrm{N}_{2}$ adsorption isotherms, using the multi-point Brunauer-Emmett-Teller (BET) method and the density functional theory (DFT) method, respectively. The $\mathrm{N}_{2}$ adsorption isotherms for the CNTs were obtained at $77 \mathrm{~K}$ (Autosorb-1, Quantachrome). Prior to the measurement of $\mathrm{N}_{2}$ adsorption, the CNTs were outgassed at $105^{\circ} \mathrm{C}$ for at least $24 \mathrm{~h}$. The morphology of the CNT aggregates was obtained using scanning electron microscopy (SEM) (SIRION-100, Fei). The contents of the oxygen-containing acidic functional groups on the CNTs were determined using Boehm's titration method [32].

\subsection{Dispersion experiments}

Twenty milliliter SDBS solutions with an initial concentration of 1000 or $5000 \mathrm{mg} / \mathrm{L}$ were mixed with $4.0,8.0$, or 12.0 $\mathrm{mg}$ of CNTs in $22 \mathrm{~mL}$ vials. The vials were sealed with aluminum foil-lined Teflon screw caps, and were sonicated at $25 \pm 5^{\circ} \mathrm{C}$ in a bath sonicator (KQ-600KDE, Shumei, China) with a fixed frequency of $40 \mathrm{kHz}$. The output power of the sonicator used in the experiments was varied to give 240, $360,420,480$ and $600 \mathrm{~W}$. The sonication time ranged from 10 to $780 \mathrm{~min}$. After sonication, the mixtures were centrifuged at $3000 \times g$ for $70 \mathrm{~min}$. The CNT suspensions (i.e. the stably suspended CNTs in aqueous phase) were then sampled to measure the concentration, and the characteristics of dispersed CNTs. Preliminary experiments indicated that the concentration of the stably suspended CNTs decreased with increasing centrifugation time, and reached a plateau at 70 min (Figure S2). The obtained CNT suspensions remained stable for at least 8 months (Figure S2). The concentration of the dispersed CNTs in the suspension was determined using UV-Vis spectrometry (UV-2450, Shimadzu) at 800 $\mathrm{nm}$ [26]. The absorbance at $800 \mathrm{~nm}$ was used to quantify the concentration $\left(C_{\mathrm{e}}, \mathrm{mg} / \mathrm{L}\right)$ in this study, because at $800 \mathrm{~nm}$, (1) the absorbance values of a CNT suspension are linearly related to the CNT concentration, as shown by the calibration curves in Figure S3; (2) no absorbance from the SDBS solution was detected; and (3) the absorbance of the CNT suspensions remained constant, even though the hydrodynamic diameter was smaller at longer sonication times (Figure S4(B)). Prior to the measurements, the CNT suspensions were diluted to a suitable concentration using ultrapure water. Our preliminary tests indicated that this dilution with ultrapure water did not affect the stabilization of the CNT suspensions (Figure S4(A)); this agreed with observations made in a previous study [33].

\subsection{Characterization of CNT suspensions}

The measurement of the hydrodynamic diameter in the CNT suspensions was performed using a Zetasizer (ZEN 3690, Malvern). Electron microscopic images of the CNT suspensions were obtained using a transmission electron microscope (TEM) (F20, Fei) with a $200 \mathrm{kV}$ electron beam, at different magnifications. A droplet of the diluted CNT suspensions was air-dried on a 300-mesh copper grid (T11012, Beijing XXBR Technology Co., Ltd.) for $2 \mathrm{~h}$ before the TEM observations.

\section{Results and discussion}

\subsection{The role of sonication time, output power, and optimal energy in the dispersion of CNTs}

Figure 1(a) shows that, within limits, the longer the sonication time, the more P-SWCNTs were dispersed in the $1000 \mathrm{mg} / \mathrm{L}$ SDBS solutions (which are identified by the dispersed concentration, $C_{\mathrm{e}}, \mathrm{mg} / \mathrm{L}$ ), and the smaller the dispersed CNT particles (identified by the average hydro-

Table 2 Selected properties of carbon nanotubes ${ }^{\text {a) }}$

\begin{tabular}{|c|c|c|c|c|c|c|c|}
\hline \multirow{2}{*}{ Notation } & \multirow{2}{*}{ Purity (\%) } & \multirow{2}{*}{ Length $(\mu \mathrm{m})$} & \multirow{2}{*}{$O D(\mathrm{~nm})$} & \multirow{2}{*}{$I D(\mathrm{~nm})$} & \multirow{2}{*}{$A_{\text {surf }}\left(\mathrm{m}^{2} / \mathrm{g}\right)$} & \multicolumn{2}{|c|}{ Amount $_{\mathrm{FG}}(\mathrm{mmol} / \mathrm{g})$} \\
\hline & & & & & & $-\mathrm{COOH}$ & $-\mathrm{OH}$ \\
\hline P-SWCNTs & $90+$ & $5-30$ & $1-2$ & $0.8-1.6$ & 385 & 0.057 & - \\
\hline H-SWCNTs & $90+$ & $5-30$ & $1-2$ & $0.8-1.6$ & 385 & 0.103 & 0.313 \\
\hline C-SWCNTs & $90+$ & $5-30$ & $1-2$ & $0.8-1.6$ & 374 & 0.509 & 0.199 \\
\hline S-SWCNTs & $90+$ & $1-3$ & $1-2$ & $0.8-1.6$ & 351 & 0.063 & 0.007 \\
\hline MWCNT30 & $95+$ & $10-30$ & $20-30$ & $5-10$ & 136 & - & 0.012 \\
\hline MWCNT100 & $95+$ & $10-20$ & $60-100$ & $5-15$ & 80 & 0.009 & - \\
\hline
\end{tabular}

a) Data of purity, length, inner diameter $(I D)$ and outer diameter $(O D)$ of $\mathrm{CNTs}$ were provided by supplier; $A_{\text {surf }}$ specific surface area; Amount ${ }_{\mathrm{FG}}$, amounts of functional group; -, undetected. 

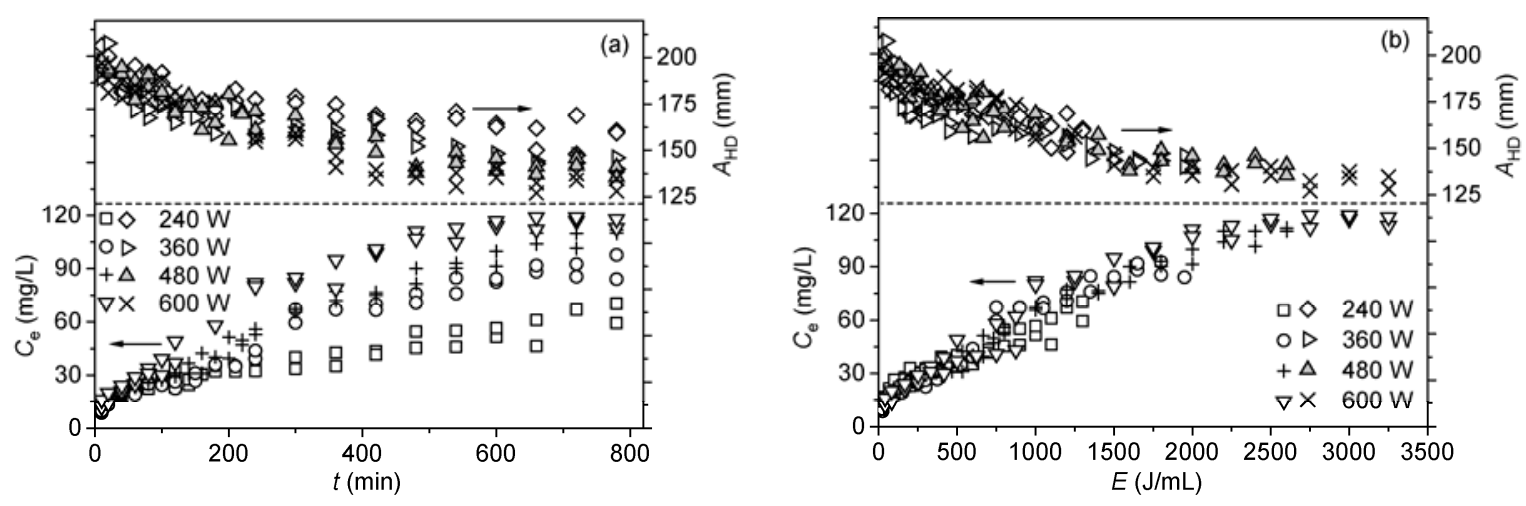

Figure 1 Stably dispersed concentration $\left(C_{\mathrm{e}}, \mathrm{mg} / \mathrm{L}\right)$ and average hydrodynamic diameter $\left(A_{\mathrm{HD}}, \mathrm{nm}\right)$ of P-SWCNTs in SDBS solution, at sonicator output powers of $240,360,480$, and $600 \mathrm{~W}$, versus sonication time $(t, \mathrm{~min})$ (a) and sonication energy $(E, \mathrm{~J} / \mathrm{mL})(\mathrm{b})$. The concentration of added P-SWCNTs and SDBS in water were 400 and $1000 \mathrm{mg} / \mathrm{L}$, respectively.

dynamic diameter, $\left.A_{\mathrm{HD}}, \mathrm{nm}\right)$ were. Both $C_{\mathrm{e}}$ and $A_{\mathrm{HD}}$ reached a plateau value under the application of additional sonication after these limits, similar to results from previous studies [7,11]. For a given sonication time, a higher output power caused more P-SWCNTs to be dispersed, and resulted in smaller $A_{\mathrm{HD}}$ values (Figure 1(a)). The four concentration curves plotted as a function of sonication energy at sonicator powers of 240,360, 480, and $600 \mathrm{~W}$ overlapped well with each other, forming a "characteristic curve" (Figure 1(b)); this was also true for the $A_{\mathrm{HD}}$ curves. $C_{\mathrm{e}}$ increased with increasing sonication energy, and reached a plateau value of approximately $110 \mathrm{mg} / \mathrm{L}$, while $A_{\mathrm{HD}}$ decreased to a plateau value of approximately $130 \mathrm{~nm}$ at an optimal energy of $2250 \pm 250 \mathrm{~J} / \mathrm{mL}$ (Figure 1(b)). The same phenomenon was also observed for the other six CNT samples, although the optimal energy values varied for the different CNT types (Figure S5). This result indicated that the dispersed concentration depended on the supplied sonication energy, but not the sonication time or the output power of sonicator alone. The fact that the sonication energy determined the dispersion of CNTs can probably be attributed to the mechanical energy provided by the sonicator overcoming the attractive vdW forces between the CNT aggregates, leading to their disentanglement and dispersion [11,34]. Another possible mechanism is the generation of cavitation bubbles in the sonication process [35-37]. When they collapse, these cavitation bubbles can form hotspots with temperatures and pressures in excess of $5000 \mathrm{~K}$ and 500 atm, respectively. These hotspots then attack the CNTs' surface or bundle end to break down the large CNT aggregates and separate the small CNT aggregates with the aid of dispersants [35-37]. More cavitation bubbles can be generated if a higher sonication energy is supplied [38].

\subsection{Influence of the SDBS and CNT concentration on the dispersion of CNT}

Figure 2(a) shows that the addition of more SDBS resulted in the dispersion of more P-SWCNTs, as indicated by the higher $C_{\mathrm{e}}$ of the P-SWCNTs dispersed in the $5000 \mathrm{mg} / \mathrm{L}$ SDBS solution compared with that of the same CNTs in the $1000 \mathrm{mg} / \mathrm{L}$ SDBS solution. However, the particle size for the P-SWCNTs dispersed in the $1000 \mathrm{mg} / \mathrm{L}$ SDBS solution was not significantly different from that measured in the $5000 \mathrm{mg} / \mathrm{L}$ SDBS solution, as indicated by the $A_{\mathrm{HD}}$ values of the dispersed P-SWCNTs (Figure 2(a)). The concentration of dispersed P-SWCNTs in the 1000 and $5000 \mathrm{mg} / \mathrm{L}$ SDBS solutions increased with increasing sonication energy, and leveled off at approximately 110 and $160 \mathrm{mg} / \mathrm{L}$, respectively (Figure 2(a)). The $A_{\mathrm{HD}}$ of the P-SWCNTs dispersed in the SDBS solutions with concentrations of 1000 and 5000 $\mathrm{mg} / \mathrm{L}$ decreased with sonication energy, until it reached a plateau value of approximately $130 \mathrm{~nm}$ (Figure 2(a)). The addition of more P-SWCNTs also resulted in a higher concentration of P-SWCNTs dispersed in the SDBS solution, but did not alter the dispersed P-SWCNT particle sizes (Figure 3(a)). At P-SWCNT concentrations of 200, 400, and $600 \mathrm{mg} / \mathrm{L}$, the concentration of dispersed P-SWCNTs in the SDBS solutions leveled off at approximately 64.0, 110, and $197 \mathrm{mg} / \mathrm{L}$, respectively (Figure 3(a)). This positive relationship between the amount of dispersed SWCNTs in the surfactant solutions and the amount of surfactants and SWCNTs added was also reported in a previous study [7]. The diameter curves for the dispersed P-SWCNTs overlapped with each other, regardless of the amount of SDBS (Figure 2(a)) or P-SWCNTs (Figure 3(a)) added, indicating that the degree of dispersion of the P-SWCNTs was independent of the amount of surfactant or CNTs added. This was consistent with the observations made by Clark et al. [39] and Wang et al. [40], which showed that the particle size was not sensitive to surfactant concentration, especially at high surfactant and SWCNT concentrations.

Although the amount of SDBS and P-SWCNTs added could alter the concentration of dispersed P-SWCNTs (Figures 2(a) and 3(a)), the optimal energy to obtain a saturated concentration $\left(C_{\mathrm{s}}, \mathrm{mg} / \mathrm{L}\right)$ of P-SWCNTs remained constant at approximately $2250 \pm 250 \mathrm{~J} / \mathrm{mL}$. Moreover, the curves showing the percentage relative suspension ratios (RSR) for 

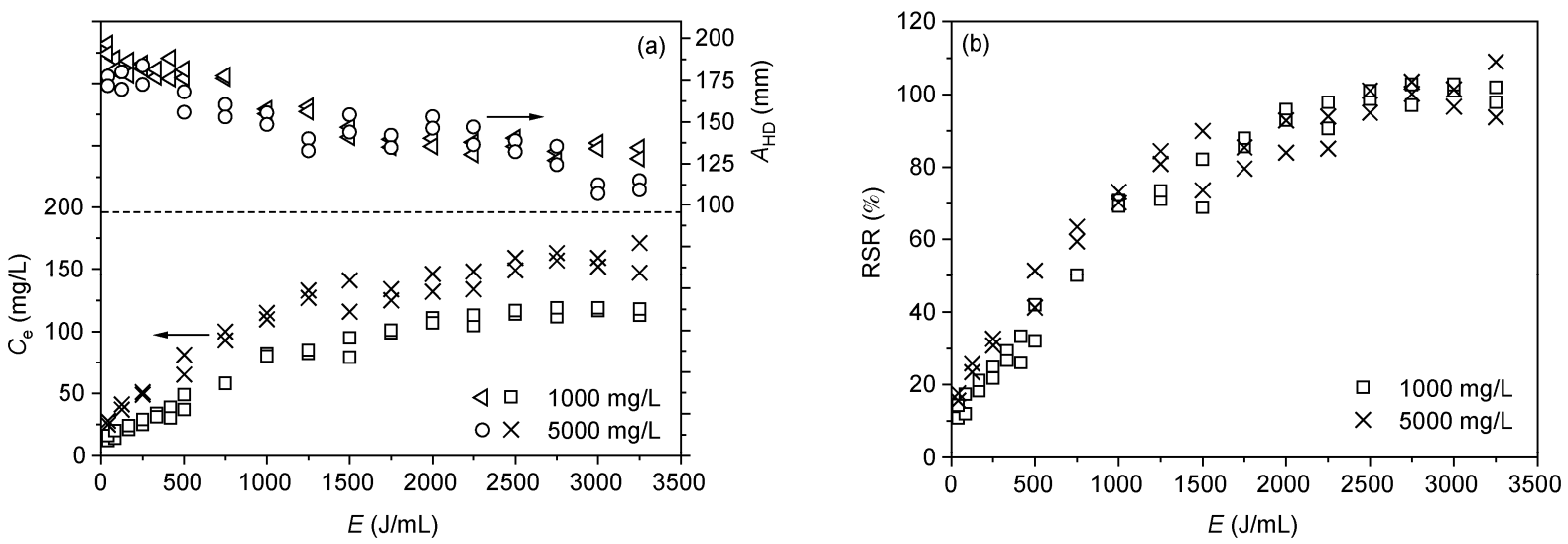

Figure 2 Stably dispersed concentration $\left(C_{\mathrm{e}}, \mathrm{mg} / \mathrm{L}\right)(\mathrm{a})$, average hydrodynamic diameter $\left(A_{\mathrm{HD}}, \mathrm{nm}\right)(\mathrm{a})$, and percentage relative suspension ratios (RSR, \%) (b) for P-SWCNTs added at a concentration of $400 \mathrm{mg} / \mathrm{L}$ to an SDBS solution with an output sonication power of $600 \mathrm{~W}$, versus the sonication energy $(E$, $\mathrm{J} / \mathrm{mL}$ ). The concentration of SDBS in water was 1000 or $5000 \mathrm{mg} / \mathrm{L}$.
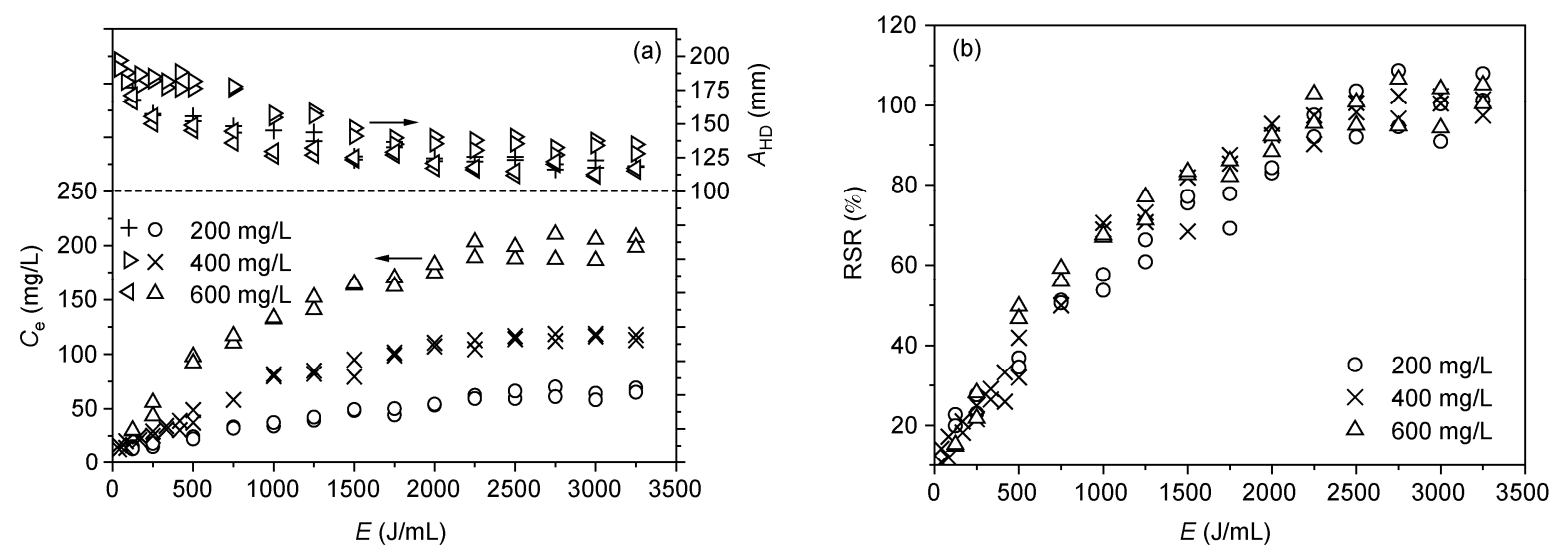

Figure 3 Stably dispersed concentration $\left(C_{\mathrm{e}}, \mathrm{mg} / \mathrm{L}\right)(\mathrm{a})$, average hydrodynamic diameter $\left(A_{\mathrm{HD}}, \mathrm{nm}\right)$ (a), and percentage relative suspension ratios (RSR, \%) (b) for P-SWCNTs in a $1000 \mathrm{mg} / \mathrm{L}$ SDBS solution with an output sonication power of $600 \mathrm{~W}$, versus the sonication energy $(E, \mathrm{~J} / \mathrm{mL})$. The concentration of P-SWCNTs in water was 200,400 or $600 \mathrm{mg} / \mathrm{L}$.

the P-SWCNTs overlapped, regardless of the amount of SDBS (Figure 2(b)) or P-SWCNTs added (Figure 3(b)), indicating that the RSR value was independent of the amount of surfactant or CNTs added. The RSR value at a given sonication energy was obtained by dividing the $C_{\mathrm{e}}$ value by the $C_{\mathrm{s}}$ value for the characteristic curves in Figures 2(a) and 3(a).

\subsection{Influence of surface functional groups, length, and outer diameter of CNTs on the dispersion}

The surface functionalization of CNTs with oxygen-containing groups (such as carboxyl functional groups) using oxidation treatment has been suggested as a potential method to promote the dispersion of CNTs in water [5]. However, the results presented in Figure 4 indicated that the promotion of the functional groups in the dispersion was insignificant if the supplied sonication energy was high enough. Although the amounts of oxygen-containing functional groups attached to the SWCNT surface increased in the order of P-SWCNTs $<$ H-SWCNTs $<$ C-SWCNTs (Ta- ble 2), the curves for the dispersed concentration and the $A_{\mathrm{HD}}$ of three SWCNTs as a function of sonication energy overlapped at relatively high sonication energies (higher than $1500 \mathrm{~J} / \mathrm{mL}$ ) (Figure 4). The plateau values of the saturated concentration $\left(C_{\mathrm{s}}, 110 \mathrm{mg} / \mathrm{L}\right)$ and $A_{\mathrm{HD}}(130 \mathrm{~nm})$ for the three SWCNTs were reached at an optimal energy of approximately $2250 \pm 250 \mathrm{~J} / \mathrm{mL}$ (Figure 4). However, a significant promotion of surface oxidation was observed in the dispersion at relatively low sonication energies (Figure 4). Surface-oxidized SWCNTs could be dispersed more easily than un-oxidized SWCNTs in the SDBS solutions; they showed higher concentrations and smaller particle sizes (Figure 4). For example, at a supplied sonication energy of $500 \mathrm{~J} / \mathrm{mL}$, the dispersed concentration of the three SWCNT types followed the order of C-SWCNTs > HSWCNTs $>$ P-SWCNTs (Figure 4), which corresponded to the amounts of attached functional groups (Table 2). This promotion effect on the CNT dispersions can be attributed to the defects on the CNT sidewalls or at the ends of sidewalls, which are generated by surface oxidation [21]; the presence of these defects means that the functionalized 
SWCNTs can be cut more easily using sonication [34]. The stronger steric repulsion between tubes that is generated by the increased amounts of functional groups was also suggested to be responsible for this promotion effect [21]. However, the former interpretation [34] may be unreasonable, because the plateau $A_{\mathrm{HD}}$ values $(\sim 130 \mathrm{~nm})$ of the three SWCNTs were almost the same (Figure 4). If the functionalized SWCNTs are more easily cut by sonication [34], the plateau $A_{\mathrm{HD}}$ value for the SWCNTs with more functional groups should be smaller. More evidence was provided by the fact that the shorter, cut SWCNTs showed lower dispersed concentrations (Figure 5(a)), but this phenomenon was not observed for the functionalized H-SWCNTs and C-SWCNTs in Figure 4. The latter interpretation [21] could also be incorrect, because the dispersed SWCNTs existed in the SDBS solutions as small aggregates, not as individual tubes (Figure 6(c)). This phenomenon, i.e. the fact that the small SWCNT aggregates were not dispersed into individual tubes by sonication, could have occurred because the low levels of supplied sonication energy were unable to overcome

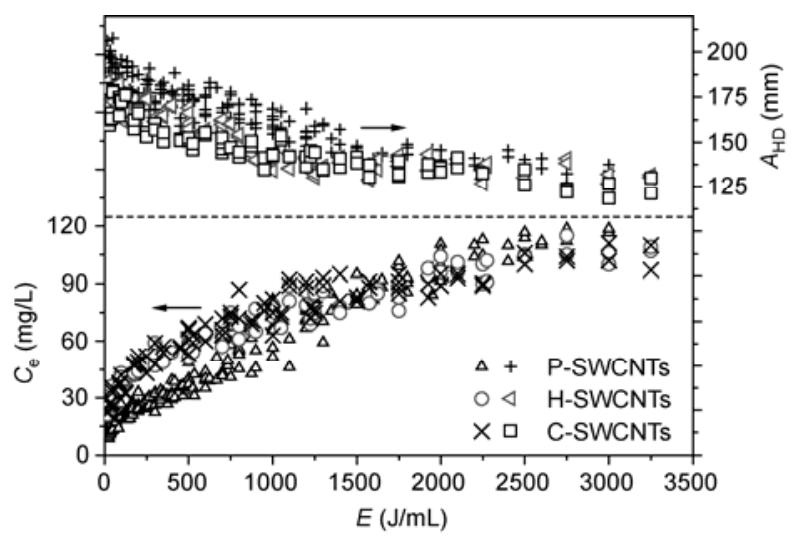

Figure 4 Stably dispersed concentration $\left(C_{\mathrm{e}}, \mathrm{mg} / \mathrm{L}\right)$ and average hydrodynamic diameter $\left(A_{\mathrm{HD}}, \mathrm{nm}\right)$ for P-SWCNTs, H-SWCNTs, and C-SWCNTs in SDBS solutions, versus sonication energy $(E, \mathrm{~J} / \mathrm{mL})$. The added concentrations of CNTs and SDBS in water were 400 and $1000 \mathrm{mg} / \mathrm{L}$, respectively. the aggregation [13]. Based on the second interpretation [21], we suggest that the promotion of surface oxidation in the SWCNT dispersions could be attributed to the stronger steric repulsion generated by the functional groups, especially between the small aggregates. Thus, the small aggregates were more easily separated from big aggregates at low sonication energies, as indicated by the smaller $A_{\mathrm{HD}}$ of the dispersed H-SWCNTs and C-SWCNTs compared with that of P-SWCNTs at very low sonication energy $(\sim 0 \mathrm{~J} / \mathrm{mL})$ (Figure 4).

The length is also thought to be one of crucial factors in the dispersion of CNTs, because longer tubes are more flexible, and are therefore more prone to entangle [14]. The longer P-SWCNTs (Table 2) showed larger $A_{\mathrm{HD}}$ values than the S-SWCNTs (which were produced using the mechanical cutting of P-SWCNTs), especially at relatively low sonication energies (Figure 5(a)). However, if the supplied sonication energy was high enough, the $A_{\mathrm{HD}}$ values of both SWCNT types decreased to the same plateau value of 130 $\mathrm{nm}$ (Figure 5(a)). The amounts of dispersed S-SWCNTs, with a plateau value of approximately $19.0 \mathrm{mg} / \mathrm{L}$, were much lower than that of the P-SWCNTs, which showed a plateau value of approximately $110 \mathrm{mg} / \mathrm{L}$ (Figure 5(a)). This difference could have been due to the fact that the S-SWCNT aggregates were smaller, stronger attractive $\mathrm{vdW}$ forces, and a higher degree of bundling than the P-SWCNT aggregates. The higher degree of bundling of the S-SWCNTs was confirmed by the smaller pore size (Figure S6) and volume $(0.427 \mathrm{~mL} / \mathrm{g}$, less than that of P-SWCNTs, $0.591 \mathrm{~mL} / \mathrm{g}$ ). In addition to the higher degree of bundling and the stronger attractive vdW forces, the smaller pore size and volume for the S-SWCNTs implied less adsorption of SDBS molecules on S-SWCNTs, and thus less dispersion. In addition, the very similar optimal energies (approximately $2250 \pm 250 \mathrm{~J} / \mathrm{mL}$, Figure 5(a) and (b)) and the overlapping of the RSR versus sonication energy curves for both SWCNTs (Figure 5(b)), indicated that these properties were independent of the length of the tubes.
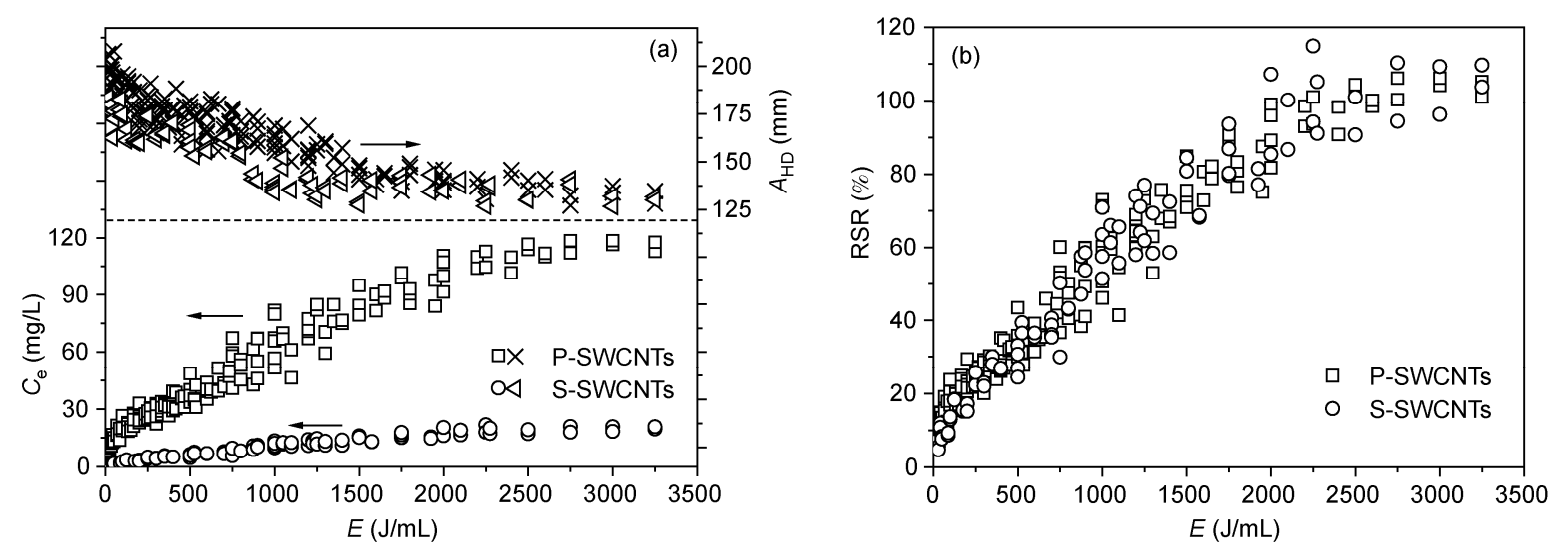

Figure 5 Stably dispersed concentration $\left(C_{\mathrm{e}}, \mathrm{mg} / \mathrm{L}\right)(\mathrm{a})$, average hydrodynamic diameter $\left(A_{\mathrm{HD}}, \mathrm{nm}\right)$ (a) and percentage relative suspension ratios (RSR, \%) (b) for P-SWCNTs and S-SWCNTs in SDBS solutions, versus sonication energy $(E, \mathrm{~J} / \mathrm{mL})$. The added concentrations of CNTs and SDBS in water were 400 and $1000 \mathrm{mg} / \mathrm{L}$, respectively. 

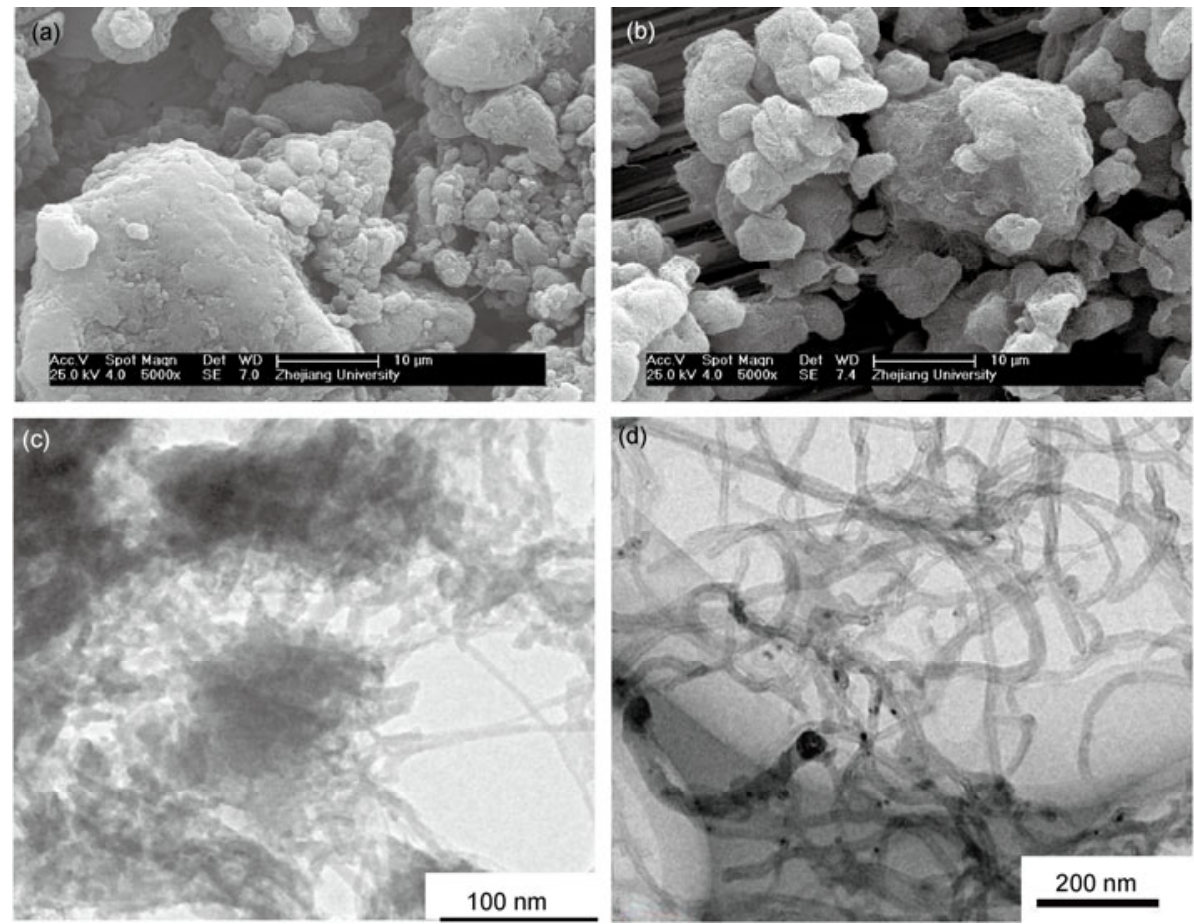

Figure 6 SEM images of un-dispersed P-SWCNTs (a) and MWCNT30 (b), as well as TEM images of stably dispersed P-SWCNTs (c) and MWCNT30 (d) The stably dispersed P-SWCNTs and MWCNT30 were prepared in $1000 \mathrm{mg} / \mathrm{L}$ SDBS solution with the sonication time of $11 \mathrm{~h}$ at the output power of $600 \mathrm{~W}$ (i.e. sonication energy of $2500 \mathrm{~J} / \mathrm{mL}$ ).

The MWCNTs (i.e. MWCNT30, MWCNT50 and MWCNT100) could be dispersed more easily than the SWCNTs in the SDBS solutions, with higher dispersed concentrations and lower optimal energy values (Figure 7). Most MWCNTs were dispersed by sonication to produce individual tubes, while the SWCNTs generally stayed as small aggregates (Figure 6(c) and (d)). This difference could be attributed to the stronger attractive vdW forces of the SWCNTs compared with those of the MWCNTs; this was supported by the fact that the aggregates of undispersed SWCNTs were larger than the aggregates of undispersed MWCNTs, as shown in the SEM images in Figure 6(a) and (b). The optimal energy values for dispersed MWCNT30, MWCNT50, and MWCNT100 were approximately $1600 \pm 150,600 \pm 150$ and $250 \pm 75 \mathrm{~J} / \mathrm{mL}$, respectively, much lower than those of the SWCNTs $(2250 \pm 250$ $\mathrm{J} / \mathrm{mL}$ ). A relationship was observed between the optimal energy values and the outer diameters (ODs, listed in Table 2) of the four CNTs (Figure 7(c)). This relationship was described by an exponential decay curve with the following equation:

$$
\begin{aligned}
\text { Optimal energy }= & (2570 \pm 363) \times \exp (-O D /(46.2 \pm 16.7)) \\
& -(210 \pm 388) \quad R^{2}=0.973
\end{aligned}
$$

This relationship indicated that CNTs with a larger outer diameter had weaker attractive vdW forces, and were thus dispersed more easily [14]. Moreover, the data for the supplied sonication energy above the exponential decay curve (Figure 7(c)) implied that excessive sonication energy was supplied to the CNT dispersion. For example, $50400 \mathrm{~J} / \mathrm{mL}$, a value approximately 20 times higher than the optimal energy for the SWCNTs $(2250 \pm 250 \mathrm{~J} / \mathrm{mL})$, was supplied for SWCNTs in a previous study [24]. Meanwhile, the data for the supplied sonication energy below the curve (Figure 7(c)) implied that in this case, the CNTs had not received enough energy to achieve a saturated suspension. In addition, the high levels of dispersion of MWCNTs observed even at very low sonication energies (Figure 7) suggested that they could move further and for longer in the environment than expected, and could therefore pose greater environment risks than SWCNTs [25,26].

\section{Conclusions}

The results obtained in this study showed that the amount of CNTs dispersed in SDBS solutions depended on the sonication energy, but not the sonication time or output power of the sonicator alone. The addition of more SDBS or CNTs resulted in the dispersion of more CNTs, while shorter or smaller tubes led to less CNT dispersion. The promotion of the surface oxygen-containing functional groups on the CNTs in the CNT dispersion was observed at relatively low sonication energies, while this promotion disappeared if the amount of supplied energy was high enough. The RSR curves for the CNT in SDBS solutions, which were plotted as a function of sonication energy, overlapped to give one curve, regardless of the amounts of SDBS or CNTs added, 

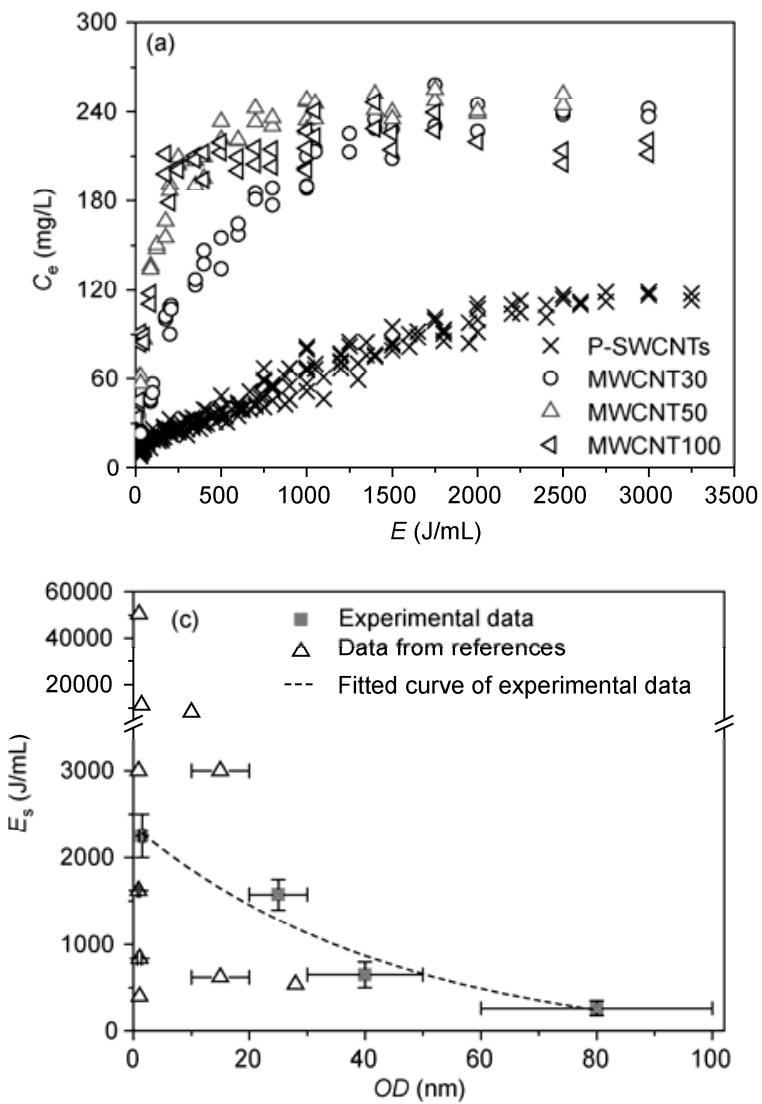

or the length of the CNTs. The optimal energy was CNT diameter-dependent, which could be attributed to the decrease in the attractive vdW forces between the CNTs produced by increases in the $O D$. However, the optimal energy was independent of the amount of surfactant or CNTs added, the length of the CNTs, and the surface functional groups of the CNTs. The exponential decay curve for the optimal energy values as a function of the outer CNT diameter could be employed to assess the use of supplied sonication energy for CNT dispersions. These results are important for the effective use of energy in industrial CNT applications.

This work was supported by the Zhejiang Provincial Natural Science Foundation of China (R5110004), the National Natural Science Foundation of China (21137003 and 40973065), the National Science \& Technology Pillar Program of China (2013BAC01B01), the Scholarship Award for Excellent Doctoral Student Granted by Ministry of Education, the Fundamental Research Funds for the Central Universities and the Key Innovation Team for Science and Technology of Zhejiang Province (2009R50047).

1 Baughman R H, Zakhidov A A, de Heer W A. Carbon nanotubesThe route toward applications. Science, 2002, 297: 787-792

2 Huang J Q, Zhang Q, Zhao M Q, et al. A review of the large-scale production of carbon nanotubes: The practice of nanoscale process engineering. Chin Sci Bull, 2012, 57: 157-166

3 Vaisman L, Wagner H D, Marom G. The role of surfactants in dispersion of carbon nanotubes. Adv Colloid Interf, 2006, 128: 37-46

4 Krause B, Villmow T, Boldt R, et al. Influence of dry grinding in a ball mill on the length of multiwalled carbon nanotubes and their dispersion and percolation behavior in melt mixed polycarbonate composites. Comp Sci Technol, 2011, 71: 1145-1153

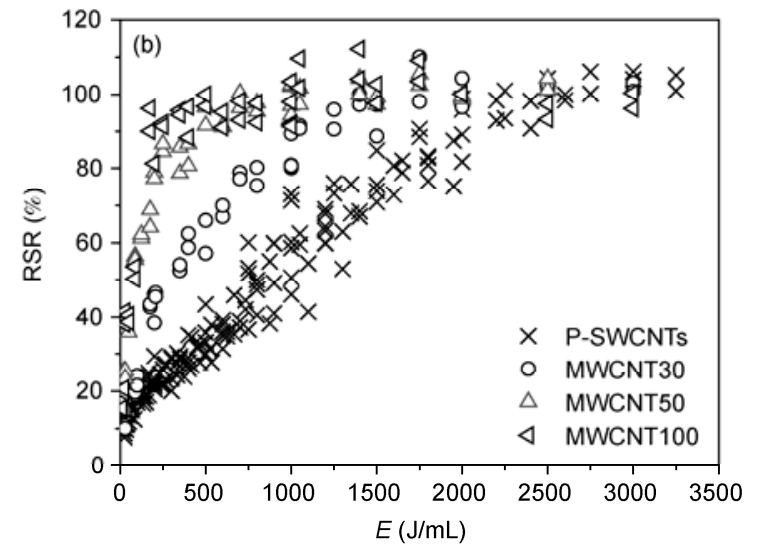

Figure 7 Stably dispersed concentration $\left(C_{\mathrm{e}}, \mathrm{mg} / \mathrm{L}\right)(\mathrm{a})$ and percentage relative suspension ratios $(\mathrm{RSR}, \%)(\mathrm{b})$ for P-SWCNTs, MWCNT30, MWCNT50, and MWCNT100 in SDBS solutions, versus sonication energy $(E, \mathrm{~J} / \mathrm{mL})$. The added concentrations of CNTs and SDBS in water were 400 and $1000 \mathrm{mg} / \mathrm{L}$, respectively. The optimal energy $\left(E_{\mathrm{s}}\right)$ for the CNTs in surfactant solution versus the CNT outer diameter $(O D, \mathrm{~nm})(\mathrm{c})$. The experimental data for P-SWCNTs, H-SWCNTs, C-SWCNTs, S-SWCNTs, MWCNT30, MWCNT50, and MWCNT100 obtained in this study was fitted using curves. The other data on the supplied energy and CNT outer diameter shown in the figure were taken from the references listed in Table 1.

5 Yamamoto T, Noda S, Kato M. A simple and fast method to disperse long single-walled carbon nanotubes introducing few defects. Carbon, 2011, 49: 3179-3183

6 Bahr J L, Yang J P, Kosynkin D V, et al. Functionalization of carbon nanotubes by electrochemical reduction of aryl diazonium salts: A bucky paper electrode. J Am Chem Soc, 2001, 123: 6536-6542

7 Matarredona O, Rhoads H, Li Z, et al. Dispersion of single-walled carbon nanotubes in aqueous solutions of the anionic surfactant NaDDBS. J Phys Chem B, 2003, 107: 13357-13367

8 Moore V C, Strano M S, Haroz E H, et al. Individually suspended single-walled carbon nanotubes in various surfactants. Nano Lett, 2003, 3: 1379-1382

9 Chen Q, Saltiel C, Manickavasagam S, et al. Aggregation behavior of single-walled carbon nanotubes in dilute aqueous suspension. J Colloid Interf Sci, 2004, 280: 91-97

10 Islam M F, Rojas E, Bergey D M, et al. High weight fraction surfactant solubilization of single-wall carbon nanotubes in water. Nano Lett, 2003, 3: 269-273

11 Yu J, Grossiord N, Koning C E, et al. Controlling the dispersion of multi-wall carbon nanotubes in aqueous surfactant solution. Carbon, 2007, 45: 618-623

12 Garg P, Alvarado J L, Marsh C, et al. An experimental study on the effect of ultrasonication on viscosity and heat transfer performance of multi-wall carbon nanotube-based aqueous nanofluids. Int $\mathrm{J}$ Heat Mass Tran, 2009, 52: 5090-5101

13 Yang Y, Grulke E A, Zhang Z G, et al. Thermal and rheological properties of carbon nanotube-in-oil dispersions. J Appl Phys, 2006, 99: 114307

14 Hilding J, Grulke E A, Zhang Z G, et al. Dispersion of carbon nanotubes in liquids. J Disper Sci Technol, 2003, 24: 1-41

15 Han Z T, Zhang F W, Lin D H, et al. Clay minerals affect the stability of surfactant-facilitated carbon nanotube suspensions. Environ Sci Technol, 2008, 42: 6869-6875

16 Krause B, Petzold G, Pegel S, et al. Correlation of carbon nanotube dispersability in aqueous surfactant solutions and polymers. Carbon, 
2009, 47: 602-612

17 Bergin S D, Nicolosi V, Cathcart H, et al. Large populations of individual nanotubes in surfactant-based dispersions without the need for ultracentrifugation. J Phys Chem C, 2008, 112: 972-977

18 Fantini C, Cassimiro J, Peressinotto V, et al. Investigation of the light emission efficiency of single-wall carbon nanotubes wrapped with different surfactants. Chem Phys Lett, 2009, 473: 96-101

19 Angelikopoulos P, Gromoy A, Leen A, et al. Dispersing individual single-wall carbon nanotubes in aqueous surfactant solutions below the cmc. J Phys Chem C, 2010, 114: 2-9

20 O'Connell M J, Bachilo S M, Huffman C B, et al. Band gap fluorescence from individual single-walled carbon nanotubes. Science, 2002, 297: 593-596

21 Zhang Y, Bai Y H, Yan B. Functionalized carbon nanotubes for potential medicinal applications. Drug Discov Today, 2010, 15: 428-435

22 Lin D, Liu N, Yang K, et al. The effect of ionic strength and $\mathrm{pH}$ on the stability of tannic acid-facilitated carbon nanotube suspensions. Carbon, 2009, 47: 2875-2882

23 Duque J G, Parra-Vasquez A, Behabtu N, et al. Diameter-dependent solubility of single-walled carbon nanotubes. ACS Nano, 2010, 4: 3063-3072

24 Tan Y Q, Resasco D E. Dispersion of single-walled carbon nanotubes of narrow diameter distribution. J Phys Chem B, 2005, 109: 1445414460

25 Jaisi D P, Elimelech M. Single-walled carbon nanotubes exhibit limited transport in soil columns. Environ Sci Technol, 2009, 43: 91619166

26 Bai Y C, Lin D H, Wu F C, et al. Adsorption of Triton X-series surfactants and its role in stabilizing multi-walled carbon nanotube suspensions. Chemosphere, 2010, 79: 362-367

27 Lin D H, Ji J, Tian X L, et al. Environmental behavior and toxicity of engineered nanomaterials (in Chinese). Chin Sci Bull (Chin Ver), 2009, 54: 3590-3604

28 Liu Y, Chen C Y. Safety and risk assessment of nanomaterials (in Chinese). Chin Sci Bull (Chin Ver), 2011, 56: 29-35
29 Field J A, Barber L B, Thurman E M, et al. Fate of alkylbenzenesulfonates and dialkyltetralinsulfonates in sewage-contaminated groundwater. Environ Sci Technol, 1992, 26: 1140-1148

30 Blackburn M A, Waldock M J. Concentrations of alkylphenols in rivers and estuaries in England and Wales. Water Res, 1995, 29: 1623-1629

31 Yang K, Jing Q F, Wu W H, et al. Adsorption and conformation of a cationic surfactant on single-walled carbon nanotubes and their influence on naphthalene sorption. Environ Sci Technol, 2010, 44: 681-687

32 de Mesquita J P, Martelli P B, Gorgulho H. Characterization of copper adsorption on oxidized activated carbon. J Brazil Chem Soc, 2006 , 17: $1133-1143$

33 Bystrzejewski M, Huczko A, Lange H, et al. Dispersion and diameter separation of multi-wall carbon nanotubes in aqueous solutions. J Colloid Interf Sci, 2010, 345: 138-142

34 Wenseleers W, Cambre S, Culin J, et al. Effect of water filling on the electronic and vibrational resonances of carbon nanotubes: Characterizing tube opening by Raman spectroscopy. Adv Mater, 2007, 19: 2274

35 Liu J, Rinzler A G, Dai H J, et al. Fullerene pipes. Science, 1998, 280: $1253-1256$

36 Strano M S, Moore V C, Miller M K, et al. The role of surfactant adsorption during ultrasonication in the dispersion of single-walled carbon nanotubes. J Nanosci Nanotech, 2003, 3: 81-86

37 Thompson L H, Doraiswamy L K. Sonochemistry: Science and engineering. Ind Eng Chem Res, 1999, 38: 1215-1249

38 Miller N. Chemical action of sound waves on aqueous solutions. Trans Faraday Soc, 1950, 46: 546-550

39 Clark M D, Subramanian S, Krishnamoorti R. Understanding surfactant aided aqueous dispersion of multi-walled carbon nanotubes. J Colloid Interf Sci, 2011, 354: 144-151

40 Wang H, Zhou W, Ho D L, et al. Dispersing single-walled carbon nanotubes with surfactants: A small angle neutron scattering study. Nano Lett, 2004, 4: 1789-1793

Open Access This article is distributed under the terms of the Creative Commons Attribution License which permits any use, distribution, and reproduction in any medium, provided the original author(s) and source are credited.

\section{Supporting Information}

Figure S1 SEM image of SWCNTs (A), MWCNT30 (B), MWCNT50 (C), MWCNT100 (D).

Figure S2 Stably dispersed concentrations $\left(C_{\mathrm{e}}, \mathrm{mg} / \mathrm{L}\right)$ of P-SWCNTs in SDBS solution versus the centrifugation time ( $t$, min) (left), visual examination of the influence of SDBS concentrations on the stabilization of P-SWCNTs (right). The stock solution was prepared by sonicating P-SWCNT-SDBS mixture at the output power of $600 \mathrm{~W}$ for $1 \mathrm{~h}$ (left) and $11 \mathrm{~h}$ (right) respectively. The added dose of P-SWCNTs and SDBS in water were 400 and 1000 or $5000 \mathrm{mg} / \mathrm{L}$, respectively.

Figure S3 The calibration curve of SWCNTs, MWCNT30, MWCNT50 and MWCNT100, i.e., stably dispersed concentrations $\left(C_{\mathrm{e}}, \mathrm{mg} / \mathrm{L}\right)$ of CNTs in SDBS solution versus the absorbance at $800 \mathrm{~nm}\left(A_{800}\right)$

Figure S4 The average hydrodynamic diameters $\left(A_{\mathrm{HD}}, \mathrm{nm}\right)$ of diluted P-SWCNTs suspensions in SDBS solution versus the standing time $(t$, min) $(\mathrm{A})$, and the absorbance at $800 \mathrm{~nm}\left(A_{800}\right)$ and the $A_{\mathrm{HD}}$ of dispersed CNTs aqueous solution versus the sonication time (B)

Figure S5 Stably dispersed concentrations $\left(C_{\mathrm{e}}, \mathrm{mg} / \mathrm{L}\right)$ and average hydrodynamic diameter $\left(A_{\mathrm{HD}}, \mathrm{nm}\right)$ of CNTs in SDBS solution at the output sonication power of 240,420 and $600 \mathrm{~W}$ versus and optimal energy $(E, \mathrm{~J} / \mathrm{mL})$. The added dose of CNTs and SDBS in water were 400 and 1000 $\mathrm{mg} / \mathrm{L}$, respectively.

Figure S6 Pore size distribution of the P-SWCNTs and S-SWCNTs obtained from the adsorption isotherms of $\mathrm{N}_{2}$ by the Density-Functional-Theory (DFT) method.

The supporting information is available online at csb.scichina.com and www.springerlink.com. The supporting materials are published as submitted, without typesetting or editing. The responsibility for scientific accuracy and content remains entirely with the authors. 